\section{The child is father of the man: the importance of early life influences on lung development}

\author{
A John Henderson
}

There is a great deal of interest in the early life determinants of lung function as possible determinants of obstructive airways disease through the rest of the life course. Failure to attain maximal lung function in early adult life either alone or coupled with accelerated decline during adulthood could contribute to the substantial burden of morbidity and mortality associated with obstructive airway disease and COPD in later life. ${ }^{1}$ Longitudinal studies of lung function in unselected populations have suggested that decrements of $\mathrm{FEV}_{1}$ established in the first few years after birth track to adulthood, ${ }^{2}$ thus pointing to early childhood as a critical period in lung function development. In this context, longitudinal cohort studies of populations that have lung function measurements during this critical period are of particular interest. Turner and colleagues report results from one of these, a birth cohort established in Perth, Western Australia, in 1987-1990 with repeat measures of lung function from shortly after birth to 18 years of age. ${ }^{3}$ The authors investigated whether factors associated with wheezing illnesses in childhood were associated with altered trajectories of lung function to age 18 years. The major influences identified were maternal asthma, atopy during infancy and maternal smoking when pregnant.

Infant lung function was measured in this cohort at three times during the first 12 months after birth and subsequent spirometry was repeated on three further occasions at 1 and 18 years. Due to its longevity, the Perth infant study used $\dot{\mathrm{V}}_{\text {max }}$ FRC rather than a raised volume method to measure infant lung function. Therefore, $\mathrm{FEF}_{25-75}$, a notoriously variable measurement, was selected as the most comparable measure from later spirometric variables. The authors also presented data using $\mathrm{FEV}_{1}$ and FVC as the later markers of lung function trajectory and provide results in a similar direction but of smaller magnitude. However,

Correspondence to Professor A John Henderson, School of Social and Community Medicine, University of Bristol, Oakfield House, Oakfield Grove, Bristol BS8 2BN, UK; a.j.henderson@bristol.ac.uk although there will inevitably be some bias in the retention of subjects available for repeat lung function measurements in later childhood, the limitations of $\mathrm{FEF}_{25-75}$ were more likely to obscure differences between groups than to introduce spurious associations. The authors have taken appropriate care to account for missingness in their sample and the consistency of associations of the key influences on lung function trajectory across different outcome measures supports the veracity of these findings.

The Tucson Children's Respiratory Study first drew attention to a relationship between lung function in early infancy and wheezing illnesses in childhood. ${ }^{4}$ The seminal findings of this cohort study suggested that transient early wheezing that resolved by school age was associated with low lung function in presymptomatic infants shortly after birth but this regressed toward the non-wheezing control values by 6 years of age. Conversely, children who developed persistent wheezing in the early years strongly associated with later asthma, lung function in infancy that was indistinguishable from controls but was significantly lower by age 6 years, inferring a lower trajectory of airway growth. This suggested that transient early wheeze was related to airway developmental disorder, whereas persistent wheezing, perhaps through remodelling of the airways, led to progressive decrements. Subsequent follow-up of this cohort to adulthood has shown that infants in the lowest quartile of $\dot{\mathrm{V}}_{\max }$ FRC measurements at around 2 months of age also had lower $\mathrm{FEV}_{1}$, $\mathrm{FEV}_{1} / \mathrm{FVC}$ and $\mathrm{FEF}_{25-75}$ at the age of 22 years and this was independent of wheezing. ${ }^{2}$ Turner and colleagues recognised a discrete group of infants in the Perth cohort on the basis of their infant lung function at 1 month, which was flow limited at FRC (rapid thoracic compression did not elevate expiratory flow above tidal flow at FRC). This could be regarded as an extreme form of airway developmental disorder and therefore might be expected to be associated with persistently abnormal airway function throughout childhood. However, in the current study and as previously reported at the age of
11 years, this particular form of airway obstruction apparent just after birth seems to resolve during childhood. ${ }^{5}$ Therefore, it would appear to behave differently from the low $\dot{\mathrm{V}}_{\text {max }}$ FRC group described in Tucson, although the reasons for this are not immediately apparent.

One of the key findings that marks this study out from a number of others was the reported absence of an association between asthma history and a low trajectory of lung function accrual during childhood, although it will be apparent that there is a negative relationship between the two and the small numbers raise the possibility of type II error. The relationship between asthma and lung function is clearly complex and potentially bidirectional. Low lung function measures in infancy before the onset of wheezing symptoms have been associated with subsequent asthma development in later childhood. Using a tidal breathing method $\left(t_{\text {ptef }} / t_{\mathrm{E}}\right)$, Håland and others reported that those infants with values below the median were more likely to have asthma at 10 years. ${ }^{6}$ Bisgaard et $a l^{7}$ measured infant lung function using a raised volume, forced expiratory manoeuvre and reported similar findings of increased asthma risk associated with evidence of airway obstruction in infancy. Furthermore, unlike Turner and colleagues' current report, the decrement in forced expiratory flows during early infancy increased over time in those subjects who developed symptoms of asthma by age 7 years. Airway remodelling with thickening of the respiratory basement membrane (RBM) is a recognised feature of moderate and severe asthma and RBM thickening has also been described in preschool wheezing in association with eosinophilic airway inflammation. ${ }^{8}$ Severe asthma in childhood ${ }^{9}$ and persistent wheezing ${ }^{10}$ have both been associated with lower lung function in longitudinal studies of asthma followed to adulthood. Therefore, there is at least some evidence that asthma in children is more likely in those with abnormal airway development, as evidenced by low lung function near birth, and that asthma may impose further decrements in lung function during childhood, perhaps through remodelling of the airways or through some other factor common to both asthma and lung development.

Early allergic sensitisation was identified in the present study as an association of lower lung function growth trajectory through childhood. A similar finding has been reported previously in the German Multicentre Allergy Study, ${ }^{11}$ the authors concluding that early sensitisation and 
subsequent chronic exposure to perennial allergens could be responsible for loss of lung function. This is consistent with observations from the Manchester Asthma and Allergy Study, where it was found that only a combination of specific sensitisation and exposure to the sensitising allergen in infancy rather than either of these factors alone was associated with subsequently poorer lung function at age 3 years. ${ }^{12}$ The mechanisms of this association are presently unclear although it is tempting to speculate that they are likely to operate through persistence of airway epithelial inflammation with adverse impacts on growth and repair processes. ${ }^{13}$ However, it is difficult to reconcile persisting airway inflammation with the absence of evidence of a diagnosis of asthma ever in the Perth cohort reported here. Additionally, the strong association of maternal asthma history with children's lung development calls for speculation about the presence of some 'Factor X' (maternal genes interacting with an unknown intrauterine influence) rather than the likely correlation between maternal and child asthma that would provide a highly plausible explanation for childhood airway inflammation and adverse effects on subsequent lung function.

COPD is an important public health problem with the World Health Organization predicting deaths associated with COPD are likely to increase by up to $30 \%$ over the next decade. Although tobacco control remains the single most important intervention, attention to the mechanisms of lung function development and its aberrations in childhood will identify factors that influence lung growth during this critical period. Associations of some early childhood factors with adult obstructive airways disease, such as low birth weight $^{14} 15$ and early childhood infections, ${ }^{16}$ have been recognised for a considerable time. The challenge is to understand the mechanisms by which exposures in early life, including pregnancy, influence lung growth and development and to develop interventions to rectify the effects of adverse influences during these critical years. Studies such as the Tucson, Copenhagen and Perth longitudinal studies of infant lung function and subsequent respiratory health have pointed the way, but interventions remain some way over the horizon.

\section{Competing interests None.}

Provenance and peer review Not commissioned; externally peer reviewed.

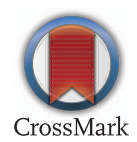

To cite Henderson AJ. Thorax 2014;69:976-977.

Published Online First 22 July 2014

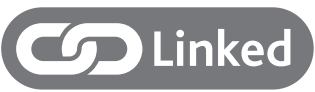

http://dx.doi.org/10.1136/thoraxjnl-2013-204931

Thorax 2014;69:976-977.

doi:10.1136/thoraxjnl-2014-205752

\section{REFERENCES}

1 Bush A. COPD: a pediatric disease. COPD 2008:5:53-67.

2 Stern DA, Morgan WJ, Wright AL, et al. Poor airway function in early infancy and lung function by age 22 years: a non-selective longitudinal cohort. Lancet 2007;470:758-64.
3 Turner S, Fielding S, Mullane D, et al. A longitudinal study of lung function from one month to 18 years of age. Thorax 2014:69:1015-20.

4 Martinez FD, Morgan WJ, Wright AL, et al. Diminished lung function as a predisposing factor for wheezing respiratory illness in infants. $N$ Eng/ I Med 1988;319:1112-17.

5 Turner SW, Palmer L, Rye PJ, et al. Infants with flow limitation at 4 weeks: outcome at 6 and 11 years. Am J Resp Crit Care Med 2002;165:1294-8.

6 Håland G, Carlsen KC, Sandvik L, et al. Reduced lung function at birth and risk of asthma at 10 years of age. N Engl J Med 2006;355:1682-9.

7 Bisgaard $\mathrm{H}$, Jensen $\mathrm{SM}$, Bonnelykke $\mathrm{K}$. Interaction between asthma and lung growth in early life. Am J Resp Crit Care Med 2012;185:1183-9.

8 Saglani S, Pyne DN, Zhu J, et al. Early detection of airway wall remodelling and eosinophilic inflammation in preschool wheezers. Am J Resp Crit Care Med 2007;176:858-64.

9 Tai A, Tran H, Roberts M, et al. Outcomes of childhood asthma to the age of 50 years. J Allergy Clin Immunol 2014;133:1572-8.

10 Sears MR, Greene JM, Willan AR, et al. A longitudinal population-based cohort study of childhood asthma followed to adulthood. N Engl J Med 2003;349:1414-22.

11 Illi S, von Mutius E, Lau S, et al. Perennial allergen sensitisation in early life and chronic asthma in children: a birth cohort study. Lancet 2006;368:763-70.

12 Lowe LA, Woodcock A, Murray CS, et al. Lung function at age 3 years: effect of pet ownership and exposure to indoor allergens. Arch Pediatr Adolesc Med 2004;158:996-1001.

13 Royce SG, Li X, Tortorella S, et al. Mechanistic insights into the contribution of epithelial damage to airway remodelling. Novel therapeutic targets for asthma. Am J Respir Cell Mol Biol 2014:50:180-92.

14 Barker J, Godfrey KM, Fall C, et al. Relation of birth weight and childhood respiratory infection to adult lung function and death from chronic obstructive airways disease. BMJ 1991;303:671-5.

15 Lawlor DA, Ebrahim S, Davey Smith G. Association of birth weight with adult lung function: findings from the British Women's Heart and Health Study and a meta-analysis. Thorax 2005;60:851-8.

16 Shaheen SO, Barker DJ, Shiell AW, et al. The relationship between pneumonia in early childhood and impaired lung function in adult life. Am J Respir Crit Care Med 1994;149:616-19. 